\title{
Models of Hysteresis in Magnetic Cores
}

\author{
A. Stancu, O. Caltun and P. Andrei \\ "Alexandru Ioan Cuza" University, Faculty of Physics, Department of Electricity, Bd. Copou 11, \\ 6600 Iasi, Romania
}

\begin{abstract}
A new method of obtaining physical information about a system of fine ferrite powder, from the hysteresis experienced by a ferromagnetic core as it dissipates energy in an RLC circuit is presented. The models of the magnetization processes are classified in three categories: physical, phenomenological and mixed. Hodgdon developed a purely mathematical model that was tested on magnetization processes in magnetic cores. The values of the model's parameters are calculated from experimental data obtained in a series RLC circuit. The capacitor discharges through the circuit and the core performs a complex magnetization process. The voltage across the capacitor and the current intensity are measured. These magnetization processes could be also simulated with a mixed-type model. The identification methodology of the mixed model requires the measurement of some magnetization curves: the first magnetization curve, the major hysteresis loop, the isothermal remanent magnetization process and the d.c. demagnetization process. In order to preserve the simplicity of the experimental data measurement and to perform the identification of the mixed model, some of the experimental curves required by the identification methodology were simulated with the Hodgdon model. Experiments were performed on soft $\mathrm{MnZn}$ ferrite core. The results of the identification are discussed.
\end{abstract}

\section{INTRODUCTION}

Anterior works by Hodgdon and co-workers [1-2] shown that the differential equation,

$$
\dot{\mathrm{H}}=\alpha \mid \dot{\mathrm{B}}[\mathrm{i}(\mathrm{B})-\mathrm{H}]+\dot{\mathrm{Bg}}(\mathrm{B}, \dot{\mathrm{B}})
$$

could be used to describe both soft and hard ferromagnetic materials magnetic processes; $\alpha$ is a constant, $f$ and $g$ are functions which must satisfy few important restriction. The functions $f$ and $g$ are piecewise smooth. The first is an odd function with a derivative having finite limit $\tilde{\mathbf{f}}^{\prime}(\infty)$; the latter is a piecewise smooth even function with the limit $\widetilde{\mathrm{g}}=\widetilde{\mathbf{f}}^{\prime}(\infty)$ and for all $\mathrm{B} \widetilde{\mathrm{g}}$ satisfy the inequality:

$$
g(B) \geq \max \left\{f^{\prime}(B), \alpha e^{\alpha B}\left|\int_{B}^{\infty}\left[f^{\prime}(\varsigma)-g(\varsigma)\right] e^{\alpha \varsigma} d \varsigma\right|\right\} .
$$

The applicability of model is proved in ferromagnetic materials, thin films used in magnetic recording established in our experiments also for ELFERRITE MnZn soft ferrite type.

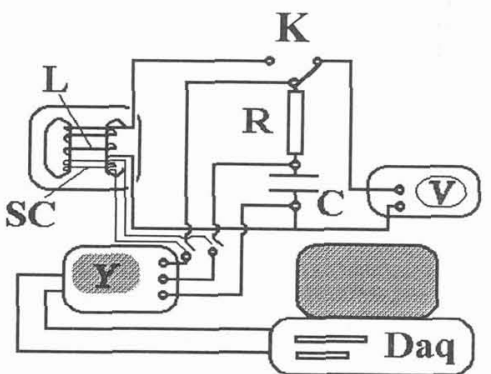

Fig. 1 Experimental set-up

\section{EXPERIMENTAL SET-UP}

Fig. 1 present the experimental setup. A RLC series circuit serves to study the magnetization processes. The inductance $L$ is a 600 turns $/ m$ coil with an $\mathrm{MnZn}$ soft ferrite core having without core $10 \mu \mathrm{H}$. The capacitance $\mathrm{C}$ $(15 \mu \mathrm{F})$ is discharged on RL circuit by a $\mathrm{K}$ switch. In our experiments on ELFERRITE core the tension on the capacitor was $70 \mathrm{~V}$. The resistance of $\mathrm{L}$ is $0.02 \Omega$ and an etalon resistance $\mathrm{R}=0.15 \Omega$ allowed to visualize a signal proportional to the current intensity. A memory digital oscilloscope was used to store the tension signals. The signal induced in a pick-up coil (SC) is proportional to the magnetic flux variation rate. By integrating with respect to the time and representing it versus field one obtains the 
hysteresis curve. The magnetic field versus time got out from the etalon resistance signal which is represented with dashed line in fig. 2.

\section{RESULTS}

The parameters respect the Hodgdon notations are shown in Table 1.

Table 1. All parameters are in MKS units

\begin{tabular}{|l|l|l|l|l|l|l|l|}
\hline $\mathrm{A}_{1}$ & $\mathrm{~A}_{2}$ & $\mathrm{~A}_{3}$ & $\mathrm{~A}_{4}$ & $\mu_{\mathrm{s}}$ & $\mu_{\mathrm{cl}}$ & $\mu_{\mathrm{c}}$ & $\mu_{\mathrm{r}}$ \\
\hline 975.7 & 4.779 & -0.569 & 0.473 & $5.510^{-6}$ & $1.0510^{-5}$ & $4.110^{-4}$ & $3.6910^{-4}$ \\
\hline $\mathrm{bp}$ & $\mathrm{B}_{\mathrm{cl}}$ & $\mathrm{B}_{\mathrm{d} 1}$ & $\mathrm{~B}_{\mathrm{d} 2}$ & $\alpha$ & $\mathrm{c}_{1}$ & $\mathrm{c}_{2}$ & \\
\hline 0.302 & 0.282 & 500 & $\infty$ & 10 & $10^{-3}$ & $210^{-5}$ \\
\hline
\end{tabular}
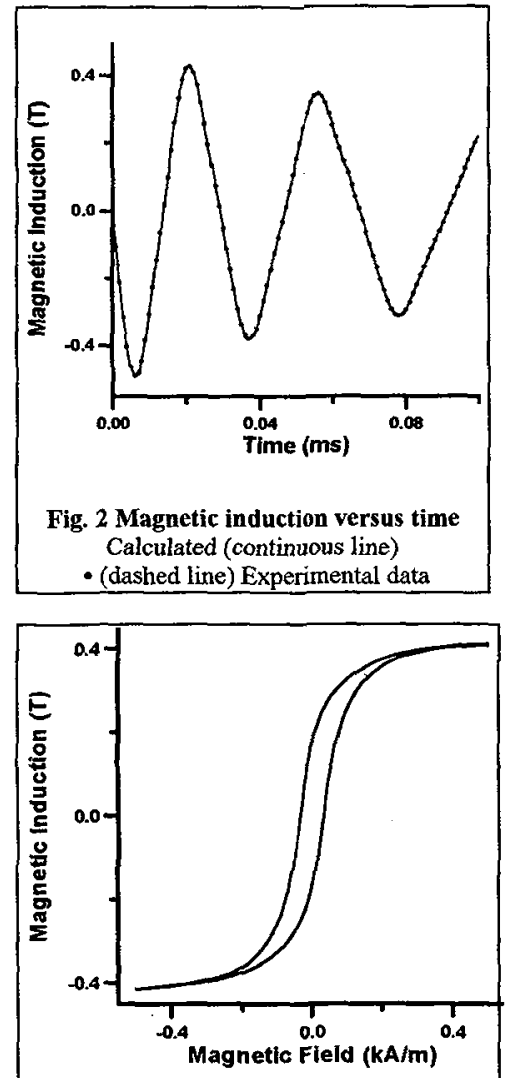

Fig.4 Calculated hysteresis loop
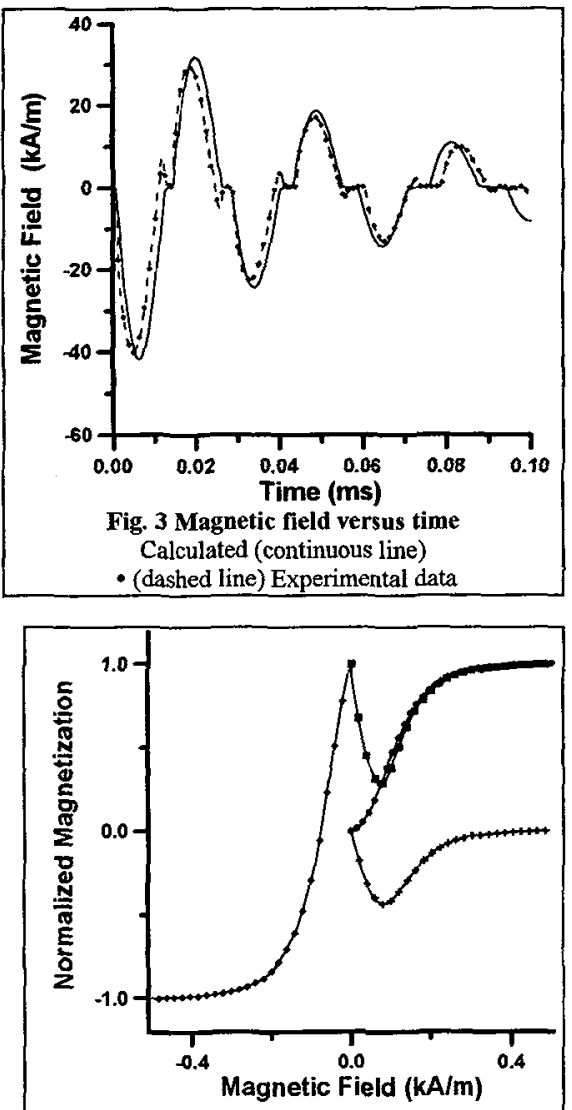

Fig. 5 Normalized IRM DCD, $\delta \mathrm{m}, \delta \mathrm{m}_{0}$ curves. - IRM; a $\delta \mathrm{mo}_{0} \rightarrow \mathrm{DCD} ;+\delta \mathrm{m}$
In the fig. 2 is presented the magnetic induction versus time. One presents both experimental and calculated magnetic field curves versus time in the fig.3. There are a good agreement between theoretical and the experimental curve.

The experimental and theoretical hysteresis loops are the same behavior. The calculated hystresis path remains very close to the major loop and in the fig. 4 one presents only the initial and major calculated magnetization curves.

The experimental data were used to find the optimal set of parameters in the Hodgdon model. With the same parameters we simulated other magnetization curves (the Isothermal Remanent Magnetization (IRM), the d.c. Demagnetization (DCD), $\delta \mathrm{m}$ curve and $\delta \mathrm{m}_{0}$ curve [3]) presented in fig. 5.

These data may be used for the parameter identification in a mixed model [4-5], which could provide physical information about the system (anysotropy field dispersion, easy axis orientation distribution, etc.). That will be the subject of another detailed paper. In this way one may obtain physical information about the sample from the experimental data in a relatively simple manner.

\section{CONCLUSION}

A new method of obtaining physical information about a ferrite sample is presented. More research has to be performed in order to verify the sensitivity of the method and the identification methodology has to be improved.

\section{References}

[1] Hodgdon, M.L., IEEE Trans. on Magn., 24(6), p.3120, 1988.

[2] Hodgdon, M.L., IEEE Trans. on Magn., 25(5), p.218, 1989.

[3] Stancu, Al., et al. "Interparticle magnetic interactions in dispersed fine particle systems" accepted to ICF-7

[4] Stancu, Al., Papusoi, Cr., Spinu, L., J.Magn.Magn.Mater., 150, p.124, 1995.

[5] Stancu, Al., presented at the MRM'95 Conference, Oxford 1995. 